Physics

Electricity \& Magnetism fields

Okayama University

Year 1992

\title{
Improvements of convergence characteristics of Newton-Raphson method for nonlinear magnetic field analysis
}

Takayoshi Nakata*

N. Okamoto**
N. Takahashi ${ }^{\dagger}$

K. Fujiwara ${ }^{\ddagger}$

Kazuhiro Muramatsu ${ }^{\dagger \dagger}$

* Okayama University

†Okayama University

$\ddagger$ Okayama University

** Okayama University

${ }^{\dagger}$ ALPS Electric Company Limited, Miyagi

This paper is posted at eScholarship@OUDIR : Okayama University Digital Information Repository.

http://escholarship.lib.okayama-u.ac.jp/electricity_and_magnetism/57 


\section{IMPROVEMENTS OF CONVERGENCE CHARACTERISTICS OF NEWTON-RAPHSON METHOD FOR NONLINEAR MAGNETIC FIELD ANALYSIS}

\author{
T.Nakata, N.Takahashi, K.Fujiwara and N.Okamoto \\ Department of Electrical Engineering \\ Okayama University \\ Okayama 700, Japan
}

\author{
K.Muramatsu \\ Central Laboratory \\ ALPS Electric Co., Ltd. \\ Kakuda 981-15, Japan
}

\begin{abstract}
In order to overcome the divergence of the Newton-Raphson iteration in the nonlinear magnetic field analysis, a relaxation factor is introduced, and its optimum value is examined. It is shown that the modified Newton-Raphson method proposed in this paper shows quick and successful convergence even in the case when the conventional Newton-Raphson method fails in convergence.
\end{abstract}

\section{INTRODUCTION}

When the magnetic scalar potential method was applied to the analysis of 3-D nonlinear magnetic fields (for example, the TEAM Workshop Problem 13), very often the nonlinear iteration using the conventional Newton-Raphson method failed to converge[1]. If the relaxation factor was introduced, the convergence characteristics were fairly improved[1]. Therefore, most codes, which employed the magnetic scalar potential method, introduced such a factor[2,3] when Problem 13 was analyzed[4].

In this paper, a method for determining the optimum relaxation factor, which utilizes the residual of Galerkin method, is developed. Moreover, some techniques to find the optimum value briefly are discussed. The effectiveness of the proposed method is illustrated quantitatively by applying the method to practical problems.

\section{MODIFIED NEWTON-RAPHSON METHOD}

\section{A. Method for Determining Optimum Relaxation Factor}

In the modified Newton-Raphson method, the obtained magnetic scalar potential $\Omega_{i}{ }^{(k+1)}$ of a node $i$ at the $(k+1)$-th iteration can be represented by the following equation :

$$
\Omega_{\mathrm{i}}^{(\mathrm{k}+1)}=\Omega_{\mathrm{i}}^{(\mathrm{k})}+\alpha^{(\mathrm{k})} \cdot \delta \Omega_{\mathrm{i}}^{(\mathrm{k})}
$$

where $\delta \Omega$ is the increment of $\Omega . \alpha$ is the relaxation factor introduced. The case of $\alpha=1$ corresponds to the conventional Newton-Raphson method. The optimum value $\alpha_{\text {opt }}$ of the relaxation factor can be determined using the linear search

Manuscript received July 7,1991 , revised October 31,1991 . This work was supported in pan by the Grant-in-Aid for Co-operative Research(A) from the Ministry of Education, Science and Culture in Japan (No.01302031) method[5].

The residual $G_{i}$ can be written as follows $[6]$ :

$$
\mathrm{G}_{\mathrm{i}}^{(\mathrm{k}+1)}=-\iiint \operatorname{grad} N_{\mathrm{i}} \cdot\left[\mu^{(\mathrm{k}+1)}\left(T_{o}-\operatorname{grad} \Omega^{(\mathrm{k}+1)}\right)\right] \mathrm{dV}(2)
$$

where $N_{i}$ is the interpolation function and $\mu$ is the permeability. $T_{o}$ is the current vector potential corresponding to the magnetizing current density. If $\Omega$ converges, $\mathrm{G}_{\mathrm{i}}$ will approach zero. Therefore, $\alpha_{\text {opt }}$ should be determined so that the following objective function $\mathrm{W}$ becomes a minimum:

$$
W^{(k+1)}=\sum_{i=1}^{n u}\left\{G_{i}^{(k+1)}\right\}^{2}
$$

where nu is the total number of unknown variables.

\section{B. Techniques for Reducing CPU Time}

Although our new method is superior from the standpoint of the convergence, the computing time for finding the optimum relaxation factor is a problem. In this section, some techniques for reducing the CPU time for finding the optimum value are discussed.

1) Calculation of objective function: The objective function $W$ defined by (3) can be separated into two parts, which are $W_{s}$ and $W_{a}$ in the nonlinear and linear regions, as follows :

$$
\begin{aligned}
& W^{(k+1)}=W_{s}^{(k+1)}+W_{a^{(k+1)}}^{(k)} \\
& W_{s}^{(k+1)}=\sum_{i=1}^{n s}\left\{G_{i}^{(k+1)}\right\}^{2} \\
& W_{a}^{(k+1)}=\sum_{j=1}^{n a}\left\{G_{j}^{(k+1)}\right\}^{2}
\end{aligned}
$$

where ns and na are the number of unknown variables in the nonlinear region and that in the linear region respectively. Since $\mathrm{W}_{\mathrm{a}}$ is negligibly small compared with $\mathrm{W}_{\mathrm{s}}$ (this will be 
shown in the next Section), the following approximation can be done:

$$
W^{(k+1)} \doteqdot W_{s}^{(k+1)}
$$

When ns is much less than na, the CPU time can be considerably saved using (7).

2) Golden section method: There are many kinds of methods to determine $\alpha_{\text {opt }}$ which gives the minimum $W_{s}$. The golden section method[5], which is a kind of the linear search method, is superior from the standpoint of the CPU time. Since the CPU time for the golden section method is dominated by the number of iterations in it, the number is discussed in the next Section.

\section{EXAMPLES OF APPLCATION}

\section{A. Analyzed Model}

As mentioned above, the TEAM Workshop Problem 13[1] which is shown in Fig.1 is chosen as the analyzed model which fails to converge by the conventional NewtonRaphson method. Since the convergence characteristics depend on the subdivision, two kinds of meshes which are shown in Fig. 2 and Table I are compared. The 1st-order brick nodal element and the $\dot{T}: \Omega$ method[6] are applied. The periodic boundary condition[1] which is applied on the $y-z$ plane enables us to analyze only $1 / 4$ of the whole region.
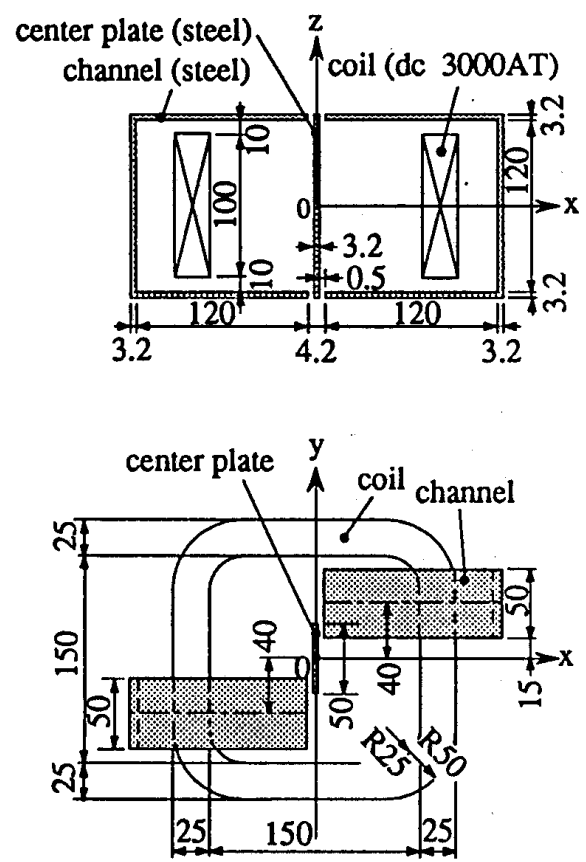

Fig. 1 3-D nonlinear magnetostatic model (TEAM Workshop Problem 13).

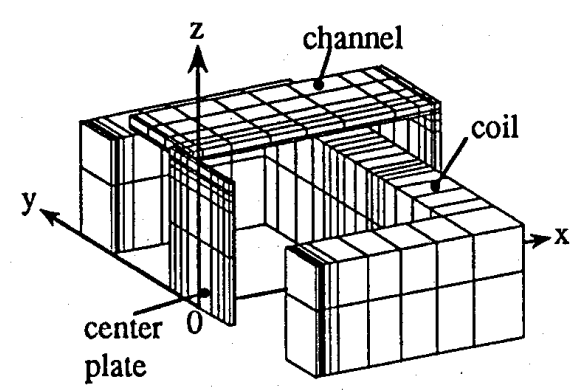

(a) coarse mesh

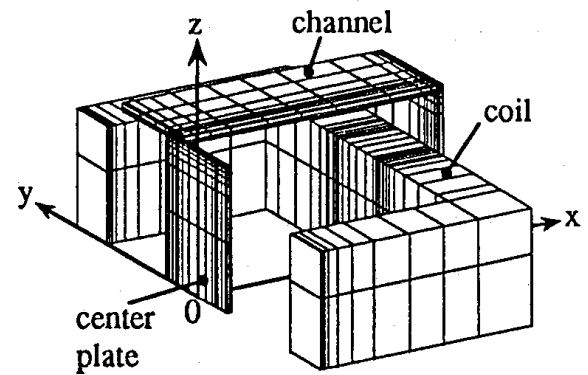

(b) fine mesh

Fig. 2 Meshes.

Table I Discretization data

\begin{tabular}{l|c|c}
\hline mesh & coarse & fine \\
\hline number of elements & 3,564 & 6,930 \\
\hline number of nodes & 4,370 & 8,184 \\
\hline number of unknowns & 2,944 & 5,950 \\
\hline number of non-zero entries & 36,012 & 74,949 \\
\hline
\end{tabular}

\section{B. Results and Discussion}

In order to examine the method for finding the optimum relaxation factor $\alpha_{\text {ope }}$, the relationship between the relaxation factor $\alpha^{(1)}$ and the objective function $W^{(2)}$ at the second step of iteration for the modified Newton-Raphson method shown in Fig. 3 is investigated. The curve for the coarse mesh has two local minima. The optimum values for the coarse and fine meshes are 0.3 and 0.2 respectively.

Figure 4 shows $\alpha_{\text {opt }}$ at each iteration for the modified Newton-Raphson method. Since $\alpha_{\text {opt }}$ changes sharply with iteration, it should be determined for every iteration.

Figures 5(a) and (b) show the objective functions $W_{s}$ and $\mathrm{W}_{\mathrm{a}}$ in the nonlinear and linear regions defined by (5) and (6) at two different steps for the modified Newton-Raphson method. $W_{\mathrm{a}}$ is negligibly small compared with $\mathrm{W}_{\mathrm{s}}$ at any step and for any $\alpha$. Therefore, the approximation using (7) is permissible.

Table II shows the numbers ns and na of unknown variables in the nonlinear and linear regions. Since ns is usually very small compared with na as shown in this table, we can use the speedup method mentioned in Sections II $B$ 1). 


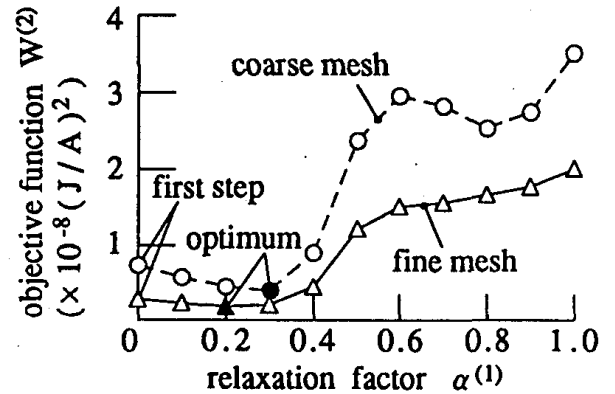

Fig. 3 Relationship between relaxation factor $\alpha$ and objective function W (second step of Newton-Raphson iteration).

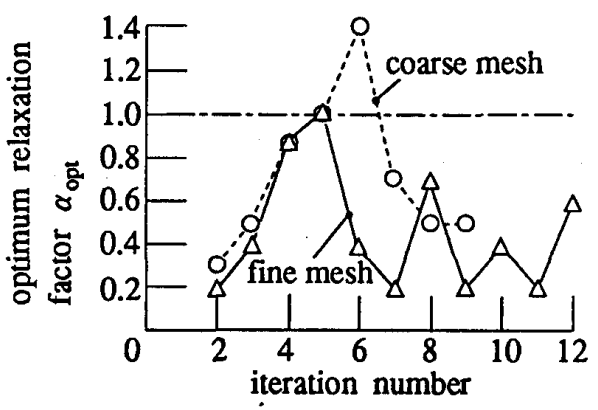

Fig. 4 Optimum relaxation factor $\alpha_{\text {opt }}$ at each iteration.

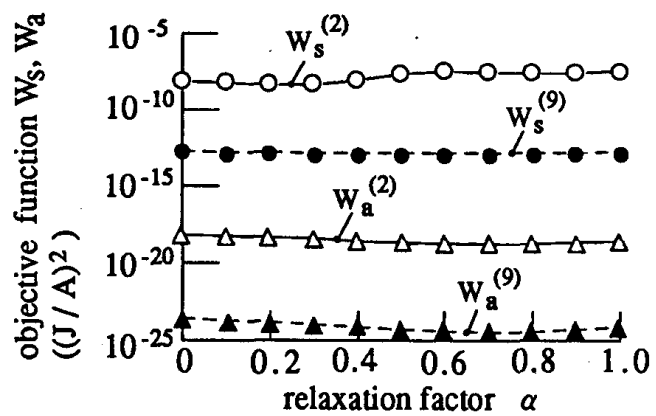

(a) coarse mesh

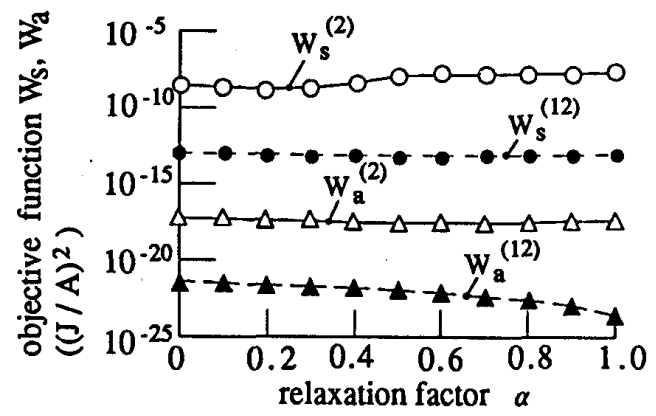

(b) fine mesh

Fig. 5 Objective functions $W_{S}$ and $W_{a}$ in nonlinear and linear regions.
Table II Number of unknown variables

\begin{tabular}{l|c|c}
\hline \multirow{2}{*}{ region } & \multicolumn{2}{|c}{ mesh } \\
\cline { 2 - 3 } & coarse & fine \\
\hline \multicolumn{1}{l|}{ total (nu) } & $2,944(1)$ & $5,950(1)$ \\
\hline nonlinear (ns) & $408(0.14)$ & $988(0.13)$ \\
\hline linear (na) & $2,536(0.86)$ & $4,962(0.87)$ \\
\hline
\end{tabular}

Figures 6(a) and (b) show the CPU time for the coarse and fine mesh respectively. The convergence criterion for the Newton-Raphson iteration is chosen as $0.01 \times \alpha \mathrm{T}$. Although $\alpha_{\text {opt }}$ may become larger than 1 as shown in Fig.4, the range of search of $\alpha$ for finding $\alpha_{\text {opt }}$ should be limited to a range between 0 and 1 . This is, because the nonlinear iteration should be decelerated by an underrelaxation factor $(\alpha<1)$. The CPU time for Newton-Raphson method does not include that for the golden section method. The CPU time for the golden section method increases considerably with the number of iterations $\mathrm{N}_{\mathrm{G}}$ for the golden section method. The total CPU time for the coarse mesh has a minimum at $N_{G}=3$. Although the suitable number of iterations for the fine mesh is not obvious due to the oscillation, $\mathrm{N}_{\mathrm{G}}=3$ can be acceptable. Therefore, $\mathrm{N}_{\mathrm{G}}$ is fixed at 3 .

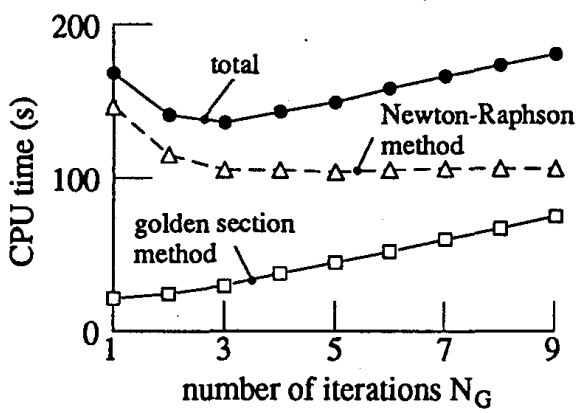

(a) coarse mesh

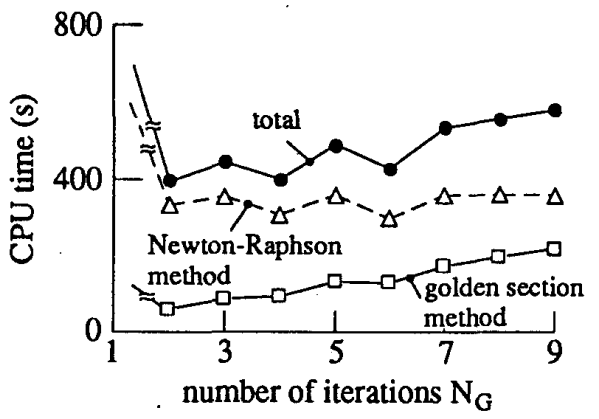

(b) fine mesh

(convergence criterion for Newton) -Raphson iteration : $0.01 \times \alpha \mathrm{T}$

Fig. 6 CPU time. 
Figure 7(a) shows the $\mathrm{x}$-component $\mathrm{B}_{\mathrm{PX}}$ of flux density at the point $P$ for the coarse mesh. The point $P$ (near the corner of the channel) is chosen from the standpoint that the flux density at the point $P$ contains remarkable error because the flux density changes steeply near here. Although the flux density oscillates when the conventional Newton-Raphson method $(\alpha=1)$ is used, it converges for the cases of $\alpha=0.5$ and $\alpha_{\text {opt }}$. Figure 7(b) shows the z-component $B_{0 Z}$ of flux density at the point $Q$ for the fine mesh. As $B_{\mathrm{PX}}$ does not oscillate violently with the number of iterations, another flux density $\mathrm{B}_{\mathrm{QZ}}$ at the point $\mathrm{Q}$ is chosen. The convergence characteristics are fairly stabilized when $\alpha_{\text {opt }}$ is used for every iteration.

Table III shows the number of iterations for NewtonRaphson method and the CPU time. Since many iterations for the golden section method are required for the

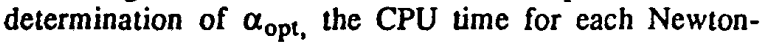
Raphson iteration is increased. However, the number of iterations for Newton-Raphson method is reduced.

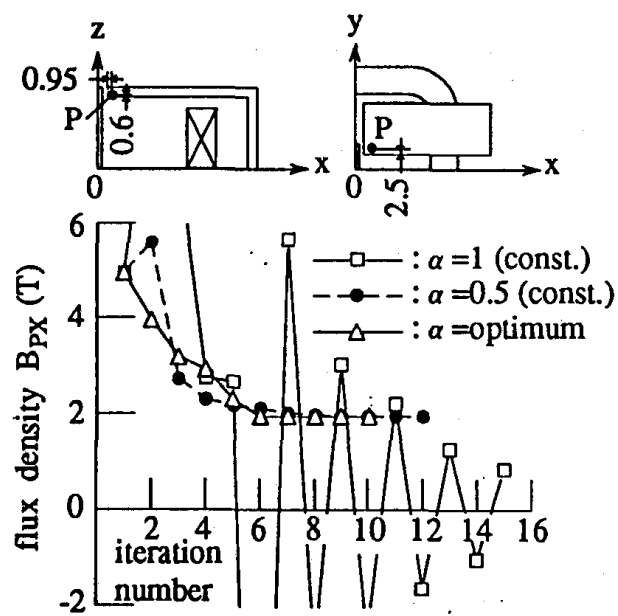

(a) coarse mesh
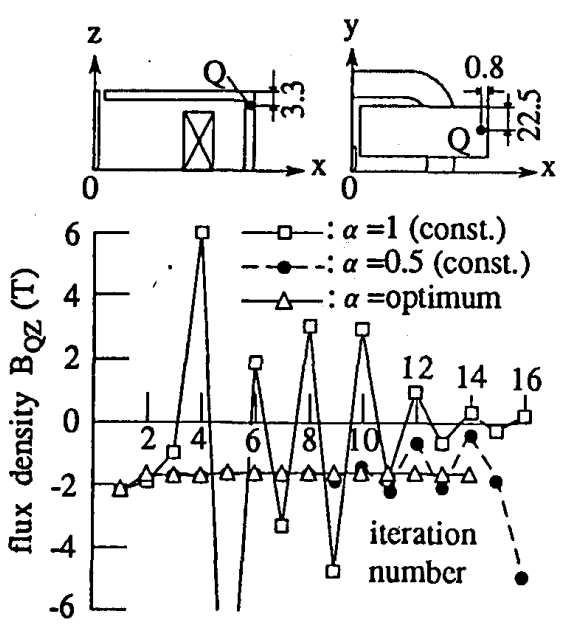

(b) fine mesh

Fig. 7 Convergence characteristics of Newton-Raphson method.
Table III Number of iterations for Newton-Raphosn method and CPU time

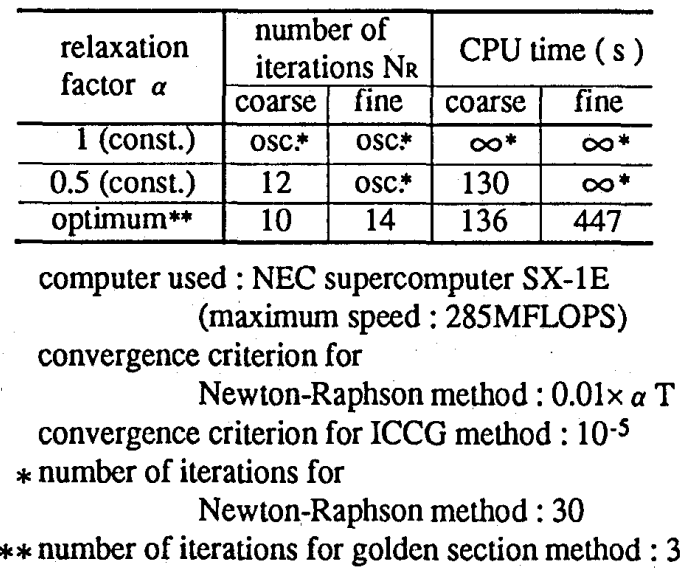

\section{CONCLUSIONS}

The results obtained can be summarized as follows:

(1) A modified Newton-Raphson method is introduced.

(2) The optimum relaxation factor can be obtained by minimizing the total square residual of Galerkin method.

(3) Methods for reducing the CPU time for finding the optimum relaxation factor are proposed.

(4) The method is applied to a practical problem and compared the convergence characteristics and the CPU time with the conventional methods.

The possibility of expansion of this method to the analysis using the magnetic vector potential will be reported in another paper.

\section{REFERENCES}

[1] T.Nakata, N.Takahashi, K.Fujiwara, K.Muramatsu and P.Olszewski : "Analysis of Magnetic Fields of 3-D Non-linear Magnctostatic Model (Problem 13)", Proceedings of the European TEAM Workshop and International Seminar on Electromagnetic Field Analysis, 107 (1990).

[2] C.A.Magele, K.Preis and W.Renhart : "Some Improvements in Nonlinear 3D Magnetostatics". IEEE Trans. Magnetics, MAG-26, 2 , 375 (1990).

[3] S.Pissanetzky : " The Interpolation of Magnetization Tables", COMPEL, 5,41 (1986) Boole Press.

[4] T.Nakata and K.Fujiwara : "Summary of Results for Benchmark Problem 13 (3-D Non-Linear Magnetostatic Model)", Proceedings of the European TEAM Workshop and International Seminar on Electromagnetic Field Analysis, 155 (1990).

[5] R.H.Gallagher and O.C.Zienkiewicz : "Optimum Structural Design -Theory and Applications-" (1973) John Wiley \& Sons.

[6] T.Nakata, N.Takahashi, K.Fujiwara and Y.Okada : "Improvements of the $T-\Omega$ Method for 3-D Eddy Current Analysis", IEEE Trans. Magnetics, MAG-24, 1, 94 (1988). 